\title{
COMPARAÇÃO DO LÉXICO DE CRIANÇAS COM SÍNDROME DE DOWN E COM DESENVOLVIMENTO TÍPICO DE MESMA IDADE MENTAL
}

\author{
Comparing the lexicon of children with Down syndrome \\ and typically developing the same mental age
}

\author{
Amanda Tragueta Ferreira(1), Dionísia Aparecida Cusin Lamônica ${ }^{(2)}$
}

\begin{abstract}
RESUMO
Objetivo: verificar o desempenho lexical, receptivo e expressivo, de crianças com Síndrome de Down e comparar com o desempenho lexical de crianças com desenvolvimento típico pareados por sexo e idade mental. Método: participaram do estudo 40 crianças, $20 \mathrm{com}$ síndrome de Down (grupo experimental - GE), de ambos os sexos, com idade cronológica variando entre 36 e 71 meses e 20 crianças com desenvolvimento típico (grupo controle - GC) pareadas quanto ao sexo e idade mental (avaliação psicológica com o instrumento Nova Versão do Método Stanford-Binet, adaptado por Terman e Merril) com o GE. Após a assinatura do Termo de Consentimento Livre e Esclarecido pelos responsáveis legais, estes responderam um protocolo de anamnese, contendo informações sobre a vida pregressa do participante. A avaliação foi composta pela aplicação do Teste de Vocabulário por Imagens Peabody (TVIP), que avalia vocabulário receptivo, e do Teste de Linguagem Infantil ABFW - Vocabulário Parte B, que avalia vocabulário expressivo. Resultados: os participantes do GE apresentaram desempenho inferior ao GC no TVIP e na designação verbal usual do ABFW, com diferenças estatisticamente significantes. Verificou-se correlação entre o desempenho no vocabulário receptivo e expressivo de ambos os grupos. Conclusão: verificou-se que o desempenho lexical, receptivo e expressivo, das crianças com SD é inferior ao de crianças com desenvolvimento típico, mesmo quando pareadas pela idade mental.
\end{abstract}

DESCRITORES: Síndrome de Down; Linguagem; Vocabulário

\section{INTRODUÇÃO}

A síndrome de Down (SD) é a causa genética mais comum de deficiência intelectual. É prevista alteração global do desenvolvimento de crianças com SD que engloba as áreas motora, cognitiva, linguística, de autocuidados e socialização ${ }^{1-8}$.

São esperadas características fenotípicas relacionadas ao comportamento de linguagem,

(1) Fonoaudióloga; Mestre em Fonoaudiologia pela Faculdade de Odontologia de Bauru da Universidade de São Paulo (FOB/USP). Bauru. São Paulo. Brasil.

(2) Fonoaudióloga; Professora Associada do Departamento de Fonoaudiologia da Faculdade de Odontologia de Bauru da Universidade de São Paulo; Livre-Docente em Distúrbios da Comunicação Humana pela Faculdade de Odontologia de Bauru da Universidade de São Paulo (FOB/USP), Bauru, São Paulo, Brasil.

Conflito de interesses: inexistente incluindo alterações na sintaxe expressiva, na emissão de morfemas gramaticais, na inteligibilidade de fala, na memória verbal, visuo-espacial e de curto-prazo e no vocabulário receptivo e expressivo $^{9-16}$. Entretanto, apesar do fenótipo das características físicas da SD ser bem descrito e fundamentado, com características linguísticas específicas previstas, a aquisição e o ritmo de desenvolvimento de linguagem de cada criança é particular.

Conforme relatado na literatura, uma característica do fenótipo comportamental de indivíduos com SD em relação ao desenvolvimento da linguagem é o seu elevado grau de variação, ou seja, relacionado à heterogeneidade do funcionamento pessoal desta casuística ${ }^{8,17-20}$.

Relacionado à forma de comunicação das crianças com SD, autores realizaram estudos enfocando diferentes objetivos quanto ao uso de gestos, considerando sua influência nas habilidades 
comunicativas e nas relações com o desenvolvimento lexical e produção verbal4,13,15,16,18,21-23.

Para Galeote et al. (2008) $)^{18}$ o desenvolvimento lexical de crianças com SD não tem sido vastamente estudado. Apresentaram que, em geral, considerase que o desenvolvimento lexical é preservado nestas crianças se comparado com outras habilidades linguísticas. Entretanto, os autores enfatizaram a importância de se considerar a relação entre o desenvolvimento cognitivo e o desenvolvimento lexical. Alguns fatores, como experiências relacionadas à idade cronológica (IC) e etiologias das alterações, podem contribuir para produzir viés na compreensão de como o desenvolvimento lexical ocorre ${ }^{24}$.

Diante o exposto, o objetivo deste estudo foi verificar o desempenho lexical, receptivo e expressivo, de crianças com SD e comparar com o desempenho lexical de crianças com desenvolvimento típico pareados por sexo e idade mental.

\section{MÉTODO}

Participaram do estudo 40 crianças, 20 com síndrome de Down (grupo experimental - GE), de ambos os sexos, com idade cronológica variando entre 36 e 71 meses e 20 crianças com desenvolvimento típico (grupo controle-GC) pareadas quanto ao sexo e idade mental com o GE.

Para o estabelecimento da idade mental os participantes foram submetidos a avaliação psicológica com o instrumento Nova Versão do Método Stanford-Binet, adaptado por Terman e Merril (1944). A dupla GE e GC foi considerada pareada quando a idade mental não ultrapassou dois meses de diferença entre o par.

A casuística deste estudo foi composta por $55 \%$ do sexo masculino e $45 \%$ do feminino. A idade mental dos participantes do GE variou de 12 a 50 meses. A idade cronológica do GC variou de 13 a 50 meses. Considerando que este grupo é de crianças típicas, a idade mental foi compatível com a idade cronológica. Todas as crianças do GE e GC frequentavam escola regular.

Após a assinatura do Termo de Consentimento Livre e Esclarecido pelos responsáveis legais, estes responderam um protocolo de anamnese, contendo informações sobre a vida pregressa do participante. A avaliação foi composta pela aplicação do Teste de Vocabulário por Imagens Peabody (TVIP) ${ }^{(25)}$, que avalia vocabulário receptivo, e do Teste de Vocabulário Infantil ABFW - Vocabulário Parte $B^{(26)}$, que avalia vocabulário expressivo.

No TVIP(25), foram seguidas as regras propostas no Manual de instruções para o estabelecimento da base e do teto das respostas. Após a aplicação do instrumento e a obtenção da pontuação de cada participante, este valor foi classificado nas categorias: baixa inferior (1), baixa superior (2), média baixa (3), média (4), média alta (5), alta inferior (6), alta (7) e alta superior (8). Quando o participante não compreendeu o teste e, assim, não obteve base, o valor considerado foi zero (0). Para esta classificação foi utilizada a idade mental.

No $A B F W^{(26)}$, os nove campos conceituais foram avaliados sempre na mesma ordem sequencial e as respostas dos participantes foram filmadas e anotadas em protocolo específico para posterior análise. Foram seguidas as regras propostas no Manual de instruções para a análise das designações dos vocábulos usuais (DVU - nomeação correta), não designações (ND - não nomeação) e processos de substituição (PS - produção de outra palavra, funcionalidade ou gesto representativo). A análise inicial constou da obtenção da média de DVU, ND e PS de cada participante, por meio da somatória das porcentagens obtidas em cada um destes itens e divisão pelo total dos campos conceituais avaliados. Para o tratamento estatístico, na realização da correlação entre procedimentos, foi utilizada a soma das porcentagens de DVU e PS (vocabulário expressivo) para correlacionar com a classificação do TVIP(25) (vocabulário receptivo).

Anteriormente à execução deste estudo o mesmo foi submetido à apreciação e aprovação (Processo no 040/2009) pelo Comitê de Ética em Pesquisa com Seres Humanos (CEP) da instituição em que o trabalho foi realizado.

O tratamento estatístico foi realizado com a aplicação de testes paramétricos e não paramétricos, de acordo com a variável estudada (Teste "t" de Student; Teste de Mann-Whitney; Coeficiente de Correlação de Spearman).

\section{RESULTADOS}

A Tabela 1 apresenta média e desvio padrão ao comparar o desempenho do GC e GE na aplicação do ABFW, por meio do teste estatístico Teste "t" Student $(p \leq 0,05)$.

A Tabela 2 apresenta média, mediana, soma de postos e posto médio ao comparar o desempenho do GC e GE na aplicação do TVIP, por meio do teste estatístico Teste de Mann Whitney $(p \leq 0,05)$.

A Tabela 3 apresenta a correlação entre o vocabulário expressivo do ABFW (DVU+PS) e o vocabulário receptivo do TVIP na amostra total e intragrupo, por meio do teste estatístico Coeficiente de Correlação de Spearman $(p \leq 0,05)$. 
Tabela 1 - Média e desvio padrão resultantes da aplicação do ABFW no GC e GE

\begin{tabular}{ccccc}
\hline ABFW & Grupo & Média & Desvio Padrão & Valor de “p” \\
\hline \multirow{2}{*}{$D V U$} & GC & 27.7 & 20.1 & \multirow{2}{*}{$0.004^{*}$} \\
& GE & 10.6 & 14.1 & 0.068 \\
\hline \multirow{2}{*}{$N D$} & GC & 24.4 & 31.7 & \multirow{2}{*}{0.691} \\
& GE & 44.6 & 36 & \\
\hline \multirow{2}{*}{$O S$} & GC & 47.9 & 19.1 & \\
& GE & 44.8 & 28.8 & \\
\hline
\end{tabular}

GC: grupo controle; GE: grupo experimental

DVU: designação verbal usual; ND: não designação; PS: processo de substituição

*: diferença estatisticamente significante

Teste estatístico: Teste " $\mathrm{t}$ " Student $(\mathrm{p} \leq 0.05)$

Tabela 2 - Média, mediana, soma de postos e posto médio resultantes da aplicação do TVIP no GC e GE

\begin{tabular}{cccccc}
\hline Grupo & Média & Mediana & Soma Postos & Posto Médio & Valor de “p” \\
\hline$G C$ & 4.4 & 5 & 495 & 24.8 & \multirow{2}{*}{$0.017^{\star}$} \\
$G E$ & 2.9 & 4 & 325 & 16.3 & \\
\hline
\end{tabular}

GC: grupo controle; GE: grupo experimental

*: diferença estatisticamente significante

Teste estatístico: Teste Mann-Withney $(\mathrm{p} \leq 0.05)$

Tabela 3 - Correlação entre vocabulário expressivo do ABFW (DVU+PS) E vocabulário receptivo do TVIP na amostra total e intra-grupo

\begin{tabular}{cccc}
\hline Grupo & Correlação & Valor de "p" & Amostra \\
\hline$G C$ & 0.63 & $0.003^{*}$ & 20 \\
$G E$ & 0.51 & $0.022^{*}$ & 20 \\
GE + GC & 0.66 & $0.000^{*}$ & 40 \\
\hline
\end{tabular}

GC: grupo controle; GE: grupo experimental

*: diferença estatisticamente significante

Teste estatístico: Coeficiente de Correlação de Spearman $(p \leq 0.05)$

\section{DISCUSSÃO}

As habilidades lexicais expressivas foram avaliadas por meio do Vocabulário Parte $\mathrm{B}^{(26)}$ do instrumento $\mathrm{ABFW}$, observando o desempenho quanto à DVU, ND e PS (Tabela 1). Verificou-se diferença estatisticamente significante apenas na DVU. Observou-se que as crianças com SD apresentaram nomeação correta, ou seja, DVU, muito abaixo do obtido pelas crianças do GC.

Quanto ao PS, as crianças com SD apresentaram porcentagens próximas às das crianças com desenvolvimento típico (DT), pois reconheceram as figuras e, dentro do seu repertório expressivo, encontraram maneiras distintas de demonstrar e expressar este conhecimento que, na maioria das vezes, não foi por meio da nomeação precisa da palavra, mas sim, com a utilização de gestos e expressões representativas.

$\mathrm{Na}$ ND as crianças com DT apresentaram médias expressivamente inferiores às crianças com SD (22.4\% para GC e $44.6 \%$ para GE). Apesar do cálculo estatístico não ter apontado diferença significante entre o desempenho dos grupos, a diferença expressiva entre eles confirma tendência das crianças com SD apresentarem padrão de comunicação inferior às crianças com DT, não nomeando maior quantidade de figuras apresentadas no teste. Infere-se que a ND pode representar que a criança não apresenta o conceito da figura, ou não reconhece a figura como representativa de um vocabulário específico, ou reconhece, mas não encontra maneira de expressar este conhecimento. 
Investigando a produção lexical de crianças com SD quanto à precisão de nomeação e o uso de gestos espontâneos frente a figuras para nomeação, a literatura apresenta que as crianças com $\mathrm{SD}$, na faixa etária pré-escolar, tendem a produzir maior número de palavras ininteligíveis e, consequentemente, maior número de gestos ${ }^{4,14}$, o que também foi verificado neste estudo.

A literatura apresenta que há diferenças significantes no número de gestos globais realizados por estas crianças e que estes gestos geralmente são produzidos acompanhados ou não por produção de fala que expressam, frequentemente, um conceito semelhante à palavra alvo, sugerindo que o conhecimento conceitual destas crianças é melhor e mais profundo do que as habilidades de expressar-se pela fala 4 .

Segundo Zampinni e D'ODorico, $(2009)^{16}$ os gestos podem fazer o papel de ponte entre a compreensão e a produção de palavras, bem como na associação entre $\mathrm{o}$ vocabulário receptivo e expressivo.

Para Galeote et al. (2008) ${ }^{18}$ crianças com SD são estimuladas em programas de intervenção precoce ao uso de gestos como parte de aquisição de habilidades comunicativas, com o intuito de melhorar suas competências interativas. Entretanto, quando se avalia a produção do vocabulário, geralmente é levada em conta somente sua modalidade expressiva oral precisa e, desta forma, a criança com $\mathrm{SD}$, que em algum momento de seu processo de aquisição usa gestos ao invés de palavras, pode apresentar desempenho inferior ao esperado para a sua competência comunicativa. Desta forma, seria conveniente para futuros estudos nesta área considerar os gestos significativos produzidos por esta criança como parte do seu desempenho lexical ${ }^{18,22,23}$.

Em relação ao vocabulário receptivo, avaliado por meio da aplicação do TVIP25 (Tabela 2), foi encontrada diferença estatisticamente significante no desempenho entre os grupos. GE apresentou média de desempenho de 2.9 e o GC de 4.4. Em outras palavras, muitos participantes com SD não compreenderam o teste (sete) e, aqueles que compreenderam, obtiveram classificação entre média baixa e média superior. Em relação ao GC, poucos participantes não compreenderam o teste (dois) e os demais apresentaram classificação entre média e alta inferior.

Ressalta-se que durante a aplicação deste instrumento foram observadas diferenças quanto ao desempenho dos grupos, ou seja, em algumas situações a criança do GE, apesar de demonstrar o conhecimento do conceito, não o reconhecia na figura, por exemplo, ao solicitar que apontasse o joelho, a criança mostrava em si, mas não era capaz de apontar a figura correta; além do uso de estratégias comunicativas não verbais, como por exemplo, pelo uso de gestos ou pantomimas representando a figura solicitada. Infere-se que o material unidimensional e em preto e branco, tenha influenciado neste comportamento, assim como o tempo de atenção para a observação de cada tela com as quatro figuras.

A literatura tem apresentado que a redução do vocabulário em indivíduos com SD pode ser decorrente do tempo de atenção diminuído, do déficit na memória de curto prazo, da memória fonológica, do atraso no desenvolvimento da função simbólica, das dificuldades para reconhecer regras gramaticais, das dificuldades na produção de fala, dos comportamentos mal-adaptativos, além de características específicas do processamento de informações auditivas e visuais nesta população $0^{1,2,6-15}$.

Quanto à correlação do desempenho dos participantes entre os testes aplicados (Tabela 3 ), verificou-se que houve correlação entre o desempenho da amostra total e de cada grupo quando comparado o desempenho no vocabulário expressivo $(A B F W)^{26}$ e receptivo (TVIP) ${ }^{25}$ e esta correlação foi estatisticamente significante. Isso significa que as crianças que apresentaram melhores desempenhos no vocabulário receptivo, também apresentaram melhores desempenhos no vocabulário expressivo, analisando-se os grupos separados ou a casuística total do estudo. O fato de esta correlação ser estatisticamente significante reforça a possibilidade, com margem pequena de erro, de extrapolar este achado para as crianças com SD e com DT em geral. O desempenho no vocabulário receptivo foi superior ao vocabulário expressivo para os dois grupos.

Em relação ao tratamento estatístico utilizado, é importante frisar que foi realizada a correlação entre os instrumentos aplicados e não a comparação, uma vez que estes apresentam seus resultados em duas medidas diferentes, ou seja, o instrumento que avalia o vocabulário expressivo utiliza medida quantitativa (porcentagem) enquanto o instrumento que avalia vocabulário receptivo utiliza medida qualitativa ordinal (ordem de classificação). Desta forma, não foi possível comparar diretamente o desempenho no vocabulário expressivo e receptivo neste estudo, apenas correlacionar o desempenho dos grupos nestas modalidades, verificando a correlação entre estas duas habilidades.

O pareamento dos grupos por idade mental resulta na média de idade cronológica do GC $(29,8$ meses) inferior ao GE (53 meses), o que pode estar relacionado aos resultados obtidos, uma vez que as experiências relativas à idade cronológica 
devem ser consideradas na compreensão de como o desenvolvimento lexical destas crianças ocorre ${ }^{24}$. Outra análise relevante refere-se ao ritmo de desenvolvimento das crianças do GC, ou seja, considerando sua faixa etária, estão cumprindo a expectativa de aquisição e desenvolvimento da linguagem. Desta forma, apresentaram o vocabulário receptivo melhor que o expressivo e com escores superiores às crianças com SD.

Vale ressaltar que na afirmação de que crianças com SD são melhores em habilidades receptivas do que em habilidades expressivas, cabe verificar quais as variáveis que estão sendo consideradas, por exemplo, Qual a faixa etária da casuística? Será considerada a IM ou a IC? Qual a metodologia? O estudo é transversal ou longitudinal? Quais instrumentos estão sendo utilizados? Em quais contextos? Diante o exposto, vários autores abordaram questões metodológicas de estudos envolvendo a $S D^{14,17,19,21,27}$ quanto à dificuldade para generalização dos dados obtidos que causam discrepâncias e dificuldades na compreensão da trajetória do desenvolvimento destes indivíduos e evidenciam a necessidade de estudos longitudinais, multidisciplinares, com grandes amostragens e utilizando procedimentos padronizados.

Os resultados deste estudo visam colaborar com a ampliação do conhecimento científico acerca do desenvolvimento linguístico das crianças com $\mathrm{SD}$, contribuindo para a reflexão sobre o desenvolvimento destas habilidades visando a compreensão destes fenômenos para dimensionar processos terapêuticos que otimizem a capacidade comunicativa destes indivíduos, contribuindo para melhorar a integração social e aprendizagem destas crianças.

\section{CONCLUSÃO}

Verificou-se que o desempenho lexical, receptivo e expressivo, das crianças com SD foi inferior ao de crianças com desenvolvimento típico, mesmo quando pareadas pela idade mental.

\section{AGRADECIMENTOS}

Apoio: Fundação de Amparo à Pesquisa do Estado de São Paulo (FAPESP) - Processo n. 2009/03355-9.

\begin{abstract}
Purpose: to check the lexical, receptive and expressive performance, of children with DS and compare it with the lexical performance of typically developing children matched for gender and mental age. Method: the study included 40 children, 20 with Down syndrome (experimental group - EG), of both genders, with age varying between 36 and 71 months and 20 typically developing children (control group - CG), matched according to gender and mental age (psychological assessment with the New Version of the Stanford-Binet method, adapted by Terman and Merrill) with EG. After the guardians have signed the consent paper, they responded to an interview protocol containing information about the previous life of the subject. The evaluation was made by applying Peabody Picture Vocabulary Test (PPVT), which assesses receptive vocabulary, and Child Language Test ABFW - Vocabulary Part B, which assesses expressive vocabulary. Results: subjects in the EG had underperformed in the GC in the PPVT and in verbal description usual in ABFW, with significant statistical differences. There was a correlation between performance on receptive and expressive vocabulary of both groups. Conclusion: it was found that the lexical, receptive and expressive performance, in children with DS is lower than in typically developing children, even when matched as for mental age.
\end{abstract}

KEYWORDS: Down Syndrome; Language; Vocabulary

\section{REFERÊNCIAS}

1. Yoder PJ, Warren SF. Early predictors of language in children with and without Down syndrome. Am J Ment Retardat. 2004;109(4):285-300.

2. Määttä $T$, Tervo-Määttä $T$, Taanila A; Kaski M, Livanainen M. Mental health, behaviour and intellectual abilities of people with Down syndrome. Down Synd Res and Pract. 2006;11(1):37-43.

3. Vicari S. Motor development and neuropsychological patterns in persons with Down syndrome. Behav Genetic. 2006;36(3):355-64.

4. Stefanini S, Caselli MC, Volterra V. Spoken and gestual production in a naming task by 
young children with Down syndrome. Brain Lang. 2007;101(3):208-21.

5. Caselli MC, Monaco L, Trasciani M, Vicari S. Language in Italian Children with Down syndrome and with Specific Language Impairment. Am Psychol Assoc. 2008;22(1):27-35.

6. Adamson LB, Bakeman RE, Deckner DF, Romski MA. Joint Engagement and the Emergence of Language in Children with Autism and Down syndrome. J Autism Dev Disord. 2009;39:84-96.

7. Rihtman T, Tekuzener E, Tenenbaum A, Bachrach $S$, Ornoy A. Are the cognitive functions of children with Down syndrome related to their participation? Dev Med Child Neurol. 2010;52(1):72-8.

8. Tsao R, Kindelberger C. Variability of cognitive development in children with Down syndrome: Relevance of good reasons for using the cluster procedure. Res Dev Disabil. 2009;30:426-32.

9. Hick RF, Botting N, Conti-Ramsden G. Short-term memory and vocabulary development in children with Down syndrome and children with specific language impairment. Develop Med \& Child Neurol. 2005;47:532-8.

10. Chapman RS. Language learning in Down syndrome: the speech and language profile compared to adolescents with cognitive impairment of unknown origin. Downs Syndr Res and Pract. 2006;10(2):61-6.

11. Jarrold C, Baddeley AD, Phillips C. Longterm memory for verbal and visual information in Down syndrome and Williams syndrome: performance on the Doors and People test. Cortex. 2007;43(2):233-47.

12. Roberts J, Price J, Barnes E, Nelson L, Burchinal M, Hennon EA, Moskowitz L, Edwards A, Malkin C, Anderson K, Misenheimer J, Hooper SR. Receptive vocabulary, expressive vocabulary, and speech production of boys with Fragile $\mathrm{X}$ syndrome in comparison to boys with Down syndrome. Am J Ment Retard. 2007;112(3):177-93.

13. Price JR, Roberts JE, Hennon EA, Berni MC, Anderson KL, Sideris J. Syntatic Complexity during conversation of boys with Fragile $\mathrm{X}$ syndrome and Down syndrome. J Speech, Lang, Hear Res. 2008;51:3-15.

http://dx.doi.org/10.1590/S1516-18462011005000041

RECEBIDO EM: 27/10/2010

ACEITO EM: 10/01/2011

Endereço para correspondência:

Dionísia Aparecida Cusin Lamônica

Via Puccini, 1-16, Residencial Tívoli I, Tívoli

Bauru - SP

CEP: 17053-095

E-mail: dionelam@uol.com.br
14. Ypsilanti A, Grouios G. Linguistic Profile of individuals with Down Syndrome: Comparing the linguistic performance of three developmental disorders. Child Neuropsychol.2008;14(1):148-70.

15. JarroldC, ThornAS, StephensE. Therelationships among verbal short-term memory, phonological awareness, and new word learning: evidence from typical development and Down syndrome. J Exp child Psychol. 2009;102(2):196-218.

16. Zampini L, D'odorico. Communicative gestures and vocabulary development in 36-month-old children with Down's syndrome. Int J Lang Comm Dis. 2009;44(6):1063-73.

17. Spanò M, Mercuri E, Randoá T, Pantoá T, Gagliano A, Henderson S, Guzzetta F. Motor and perceptual-motor competence in children with Down syndrome: variation in performance with age. Europ $\mathrm{J}$ of Paed Neurol. 1999;3:7-14.

18. Galeote M, Soto P, Checa E, Gómez A, Lamela $\mathrm{E}$. The acquisition of productive vocabulary in Spanish children with Down syndrome. J Int Dev Dis. 2008;33(4):292-302.

19. Martin GE, Klusek J, Estigarribia B, Roberts JE. Language characteristics of individuals whith Down Syndrome. Top Lang Disorders. 2009;29(2):112-32. 20. Campos AC, Rocha NACF, Savelsbergh GJP. Development of reaching and grasping skills in infants with Down syndrome. Res Dev Disabil. 2010;31:70-80.

21. Caselli MC, Vicari S, Longobardi E, Lami L, Pizolli C, Stella G. Gesture and words in early development of children with Down syndrome. Am Speech, Lang, Hear Assoc. 1998;41:1125-35.

22. Alony S, Kozulin A. Dynamic assessment of receptive language in children with Down syndrome. Adv in Speech, Lang Pathology. 2007;9(4):323-31. 23. Flabiano FC. A constituição da representação pela criança com síndrome de Down [tese]. São Paulo: Faculdade de Medicina, Universidade de São Paulo; 2010.

24. Facon B, Grubar JC, Gardez C. Chronological age and receptive vocabulary of persons with Down syndrome. Psychol Reports. 1998;82:723-6.

25. Dunn LM, Padilla ER, Lugo DE. Teste de Vocabulário em Imagens Peabody (Peabody Picture Vocabulary Test), adaptação Hispano-americana. Espanha: Circle Pines: American Guidance Service; 1986.

26. Béfi-Lopes DM. Vocabulário (Parte B). In: Andrade CRF, Béfi-Lopes DM, Fernandes FDM, Wertzner HF. ABFW - Teste de linguagem infantil: nas áreas de fonologia, vocabulário, fluência e pragmática. Carapicuíba, São Paulo: Pró-Fono, Cap. 2, 2000.

27. Groen MA, Yasin I, Laws G, Barry JG, Bishop DVM. Weak hand preference in children with Down syndrome is associated with language deficits. Dev Psycholbiol. 2008;50:242-50. 